\title{
Improved endoscopic resection of large flat lesions and early cancers using an external additional working channel (AWC): a case series
}

\section{(ㄷ)(우우}

\author{
Authors \\ Institutions \\ 1 Clinic for Internal Medicine I, Department of \\ Gastroenterology, InExEn, University Hospital UIm, Ulm, \\ Germany \\ 2 Institute of Microtechnology and Medical Device \\ Technology, Technical University of Munich, Munich, \\ Germany
}

Benjamin Walter ${ }^{1}$, Simone Schmidbaur ${ }^{1}$, Yannick Krieger ${ }^{2}$, Alexander Meining ${ }^{1}$

submitted 16.7.2018

accepted after revision $\quad 10.10 .2018$

\author{
Bibliography \\ DOI https://doi.org/10.1055/a-0824-6912 | \\ Endoscopy International Open 2019; 07: E298-E301 \\ (c) Georg Thieme Verlag KG Stuttgart · New York \\ ISSN 2364-3722
}

Corresponding author

Dr. med. Benjamin M. Walter, Uniklinik Ulm, Klinik für Innere Medizin I, Albert-Einstein-Allee 23, $89081 \mathrm{Ulm}$ Fax: +0049 731/500 44502

Benjamin.walter@uniklinik-ulm.de

\section{ABSTRACT}

Background En-bloc resection of large, flat lesions or early stages of cancer is challenging. No bimanual tasks are possible using standard endoscopes. Dual-channel endoscopes are not available everywhere and have a small distance between the channels.

Patients and methods A new external additional working channel (AWC) (Ovesco, Tuebingen, Germany) was designed and developed potentially enabling bimanual tasks. Fixed to the tip of a standard gastroscope or pediatric colonoscope, a second endoscopic tool can be inserted through the AWC and used for tissue retraction during endoscopic resection.

Results In the upper and lower gastrointestinal tract, endoscopic mucosal resection (EMR) with a modified grasp-and-snare technique and endoscopic submucosal dissection (ESD) were performed successfully using the AWC in eight patients. Complications were acute arterial bleeding post-EMR in two cases treated by endoscopic clipping.

Conclusions We conclude that a newly developed external additional working channel (AWC) enables endoscopic resection of large lesions in the upper and lower gastrointestinal tract. Potential benefits are its suitability for EMR and ESD, no need for a dual-channel endoscope and an adjustable distance of working channels.

\section{Introduction}

Endoscopic resection of lateral-spreading polyps, flat lesions larger than $2 \mathrm{~cm}$ or early stages of cancer can be challenging for interventional endoscopists. Endoscopic mucosal resection (EMR) has been widely accepted as an effective and minimally invasive treatment for patients with large gastrointestinal adenomas. Endoscopic submucosal dissection (ESD), can be considered for en-bloc resection of early cancers in the gastrointestinal tract.

For standard EMR, the lesion, augmented by submucosal injection, is removed by a through-the-scope electrocautery snare. For a large specimen, the so-called piece-meal technique (fragmented removal) is applied, which is effective and fast, but complete extraction cannot be assessed by histology. This may be a minor problem with low-grade adenomas but is essential for high-grade neoplasia and early cancers. Recent variations of the standard EMR technique include cap-assisted methods, EMR with prior band ligation of the resection area or use of a two-channel endoscope [1].

Endoscopic submucosal dissection (ESD) was first developed for en-bloc resection of early cancerous gastrointestinal lesions in Japan in the mid-1990s [2]. Currently, it is used for treatment of lesions in the upper as well as the lower gastrointestinal tract [3]. It is particularly recommended for larger specimens with no evidence of deep tissue infiltration. However, ESD is very timeconsuming, difficult to learn and has a higher risk for perforation [4]. 


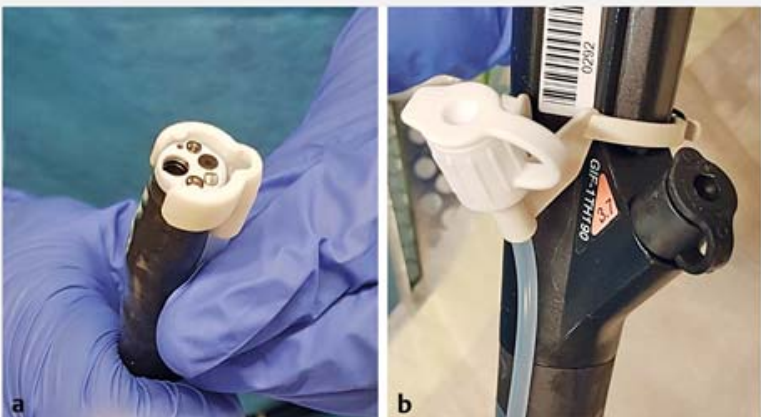

- Fig. 1 a AWC mounted on the tip of the endoscope with a freely adjustable distance to the regular working channel. b AWC-valve attached to the shaft of the endoscope.

To overcome these limitations, bimanual resection enabling traction and countertraction during resection appears helpful. This might be attempted using a dual-channel endoscope [5]. Similar to the cap-assisted method, a snare is introduced through one of the working channels of the endoscope. Grasping forceps introduced through the other channel can be used to pull the lesion into the snare loop [6]. However, the small distance between the two channels complicates the movement options and thereby reduces the effectiveness of the method.

Based on all these considerations, an additional working channel (AWC) was developed, designed and manufactured, that might potentially enable a more effective grasp-and-snare technique for EMR, as well as traction of a lesion during ESD.
Here, we present our preliminary experience of such procedures in the upper and lower gastrointestinal tract using the AWC (Ovesco, Tuebingen, Germany).

\section{Patients and methods}

To provide the advantages of dual-channel endoscopes but enlarge the distance between the channels and thereby enhance the mobility of the tools, an additional working channel (AWC) was attached to a standard endoscope ( $\mathbf{F i g . 1}$ ). It fits on endoscopes ranging from $8.5-13.5 \mathrm{~mm}$ in diameter - the fastener can be adjusted to the scope's handle ( $\triangleright$ Fig.1). The AWC can be mounted on the tip of the endoscope with a freely adjustable distance to the regular working channel, additionally stabilized by a circular tape. It is available with two different working channel lengths $(122 \mathrm{~cm}$ and $185 \mathrm{~cm})$. Tools up to $2.8 \mathrm{~mm}$ in diameter can be inserted through the AWC.

A descriptive study was performed including data on eight non-consecutive cases of patients with either indication for EMR (4 cases) or ESD (4 cases).

Individuals $>18$ years were enrolled for treatment applying the following inclusion criteria: lesion in stomach or colon with a clear indication for endoscopic removal (e.g. histology), standard EMR or ESD feasible, written consent obtained. Exclusion criteria were lack of agreement or no indication for endoscopic treatment. Descriptive statistical analysis was performed on the data.

All resections were performed using Fuji EC-760ZP-V/L, Olympus GIF-1TH190 or Olympus GIF-HQ190 endoscopes by a highly experienced interventional endoscopist. In the reported cases, the endoscopist worked with two experienced endoscopic nurses handling the tools for AWC and regular working channel.
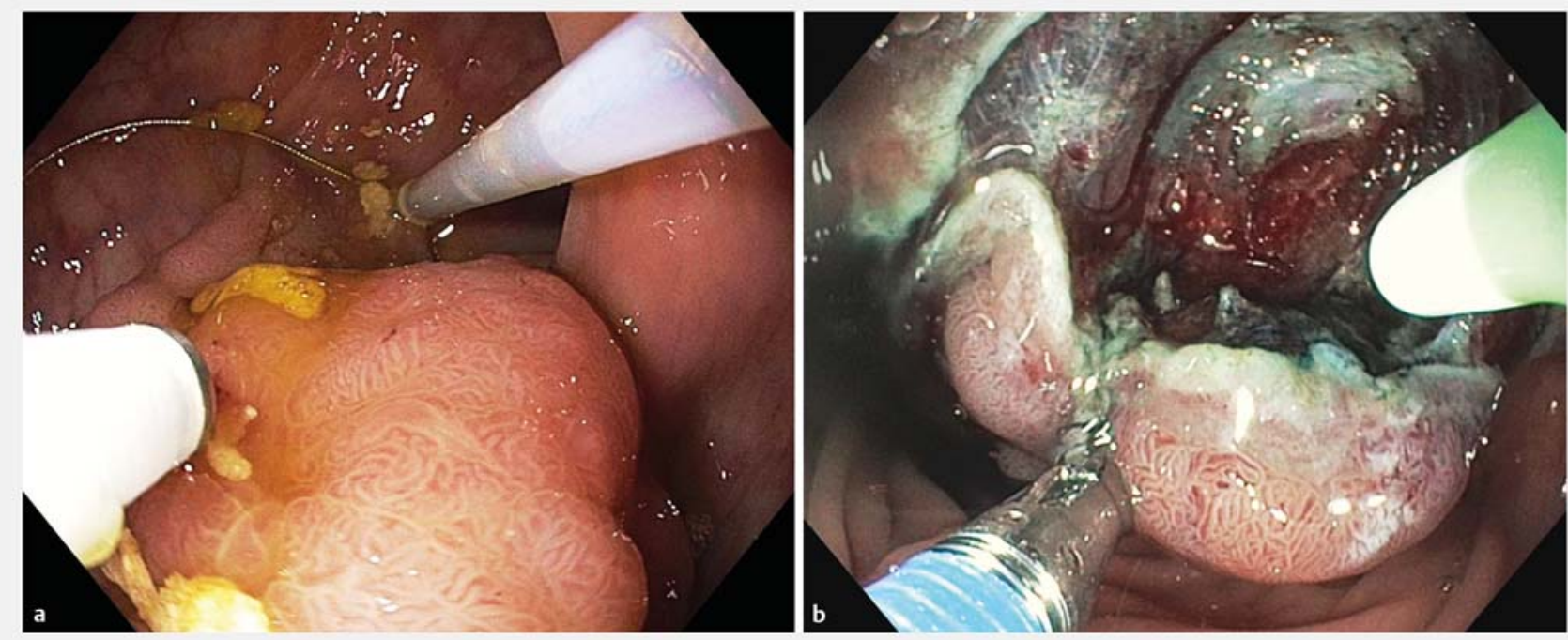

$\checkmark$ Fig. 2 a EMR with modified grasp-and-snare technique with the AWC. Electrocautery snare introduced through the additional working channel and wrapped around the base of the lesion. Injection of indigo stained saline via the regular working channel. b Endoscopic submucosal dissection. Mucosa grasped with grasping forceps introduced through biopsy channel (lower left). AqaNife introduced via the additional working channel (AWC) (upper right). 
- Table 1 Characteristics and location of resected adenomas/early stage cancer.

\begin{tabular}{|l|l|l|l|l}
\hline Technique & Location & Size (max) & Etiology & Histology/results \\
\hline EMR & Gastric posterior wall & $31 \mathrm{~mm}$ & Adenoma & Low-grade, R0 \\
\hline EMR & Ascending colon & $45 \mathrm{~mm}$ & Adenoma & Low-grade, 3 fragments \\
\hline EMR & Transverse colon & $42 \mathrm{~mm}$ & Adenoma & High-grade, R0 \\
\hline EMR & Sigmoid colon & $45 \mathrm{~mm}$ & Adenoma & Low-grade, 2 fragments \\
\hline ESD & Gastric greater curvature & $17 \mathrm{~mm}$ & Early gastric cancer & PT1a, R0, low-risk \\
\hline ESD & Gastric posterior wall & $37 \mathrm{~mm}$ & Early gastric cancer & PT1a, R0, low-risk \\
\hline ESD & Rectum & $37 \mathrm{~mm}$ & Carcinoma & PT1, R0, low-risk \\
\hline ESD & Rectum & Adenoma with focal high-grade dysplasia & High-grade, R0 \\
\hline EMR, endoscopic mucosal resection; ESD, endoscopic submucosal dissection & & \\
\end{tabular}

\section{EMR}

For modified grasp-and-snare technique using AWC for EMR, the resection area was marked with a coagulation tool, followed by a $40-\mathrm{mm}$ snare introduced through the additional working channel and wrapped around the base of the lesion. It was slightly tightened and a needle was inserted through the regular working channel. The base of the lesion was injected with indigo-stained saline. The needle was then exchanged with a tissue anchor or forceps that dragged the specimen into the loop of the snare. The electrocautery snare was closed and the specimen removed. During pre-work on EMR technique using a porcine ex-vivo model (unpublished data), it was found that gently pushing back the tissue before resection reduces risk for perforation ( $\triangleright$ Fig. 2a).

\section{ESD}

For ESD, lesions were marked by coagulation. After submucosal injection, mucosal incision was performed. Mucosa was grasped with grasping forceps introduced through the AWC or biopsy channel depending on the position and submucosal dissection was performed using the AqaNife (OVESCO, Tuebingen, Germany) or DualKnife (Olympus, Tokio, Japan) ( $\triangleright$ Fig. 2b).

\section{Results}

EMR with modified grasp-and-snare technique using AWC was successfully performed in four patients (1 upper gastrointestinal tract, 3 lower gastrointestinal tract). ESD was successfully performed in four patients ( 2 upper gastrointestinal tract, 2 lower gastrointestinal tract) with endoscopic success being defined as a technically feasible intervention.

The macroscopically estimated lesion size was $35.9 \mathrm{~mm}$ on average. Median age of treated patients was 81.0 years ( $5 \mathrm{fe}-$ male, 3 male). Mean procedure time (scope-in to scope-out) was 68.5 minutes. On average $427.1 \mathrm{mg}$ of propofol and $3.3 \mathrm{mg}$ of midazolam were administered for sedation. Reported complications were acute arterial bleeding directly post-EMR in two cases terminated by either hemoclipping alone (one case) or combination of hemoclipping, submucosal injection of adrenalin 1:10000 and hot biopsy forceps (one case).

No delayed bleeding, no perforation and no further severe adverse events occurred.

R0-resection was achieved in all of the four cases treated by ESD and in two cases treated by EMR. The remaining two cases were treated by piecemeal EMR with positive lateral margins. Early gastric cancer pT1a, Gll was diagnosed in two cases. Lowgrade adenomas were diagnosed in three out of eight cases. In one patient the pathologist reported an adenoma with focal high-grade dysplasia (R0) (> Table 1).

\section{Discussion}

Endoscopic resection of large, flat lesions can be challenging. EMR and ESD are now well-established standard techniques for interventional endoscopy. However, these techniques have certain procedural limitations. The major drawbacks are that with EMR, downgrade to piecemeal resection is often necessary, whereas ESD for en bloc resection is time consuming and has a higher risk for perforation. In addition, both techniques are hampered by the fact that using standard endoscopes only a single instrument can be operated at a time [7]. Several promising approaches were already tried for achieving an eased work setting [8]. Yet the approved devices are either only fitting for specific indications in the rectum or, as with the Endolifter (Olympus), possible manipulation of the mucosa is limited to solely retraction $[9,10]$. In certain centers, ESD is also performed by tunneling techniques or counter-traction with dental floss by clip and string technique. It has to be noted that the latter is an off-label use.

In contrast, the AWC enables introduction of an additional tool for a distinct traction and counter-traction of tissue. The device is mounted onto the tip of the endoscope at any freely chosen position at the discretion of the interventional endoscopist. The distance of the two separate working channels can be adapted to the respective intervention. In comparison to a narrower diameter of dual-channel endoscopes, a larger distance between the channels could enable the endoscopist to 
make better use of the traction and counter-traction principle and enable more effective use of leverage effect. Furthermore, in contrast to certain retraction devices, pushing and pulling of the mucosa is possible to potentially ease endoscopic resection.

However, both instruments can only be used parallel in one horizontal level. Further development of a bendable bimanual grasping device might be reasonable as a next step.

Because the AWC is an overtube fixed to the endoscope with a circular tape, further limitation might be the possibility that the additional working channel might come loose and drop away during the procedure. Last but not least, the diameter of the endoscope is increased by attachment of the AWC to an additional $3 \mathrm{~mm}$. Also, not observed so far, this might be a limitation in entering smaller lumina or passing the pharynx.

\section{Conclusion}

Nevertheless, based on our preliminary experience, we conclude that the newly developed external additional working channel enables endoscopic resection of large lesions in the upper and lower gastrointestinal tract. It has the potential to at least partly overcome current limitations of endoscopic resection. Further data on its broader application is desirable.

\section{Acknowledgement}

This work was supported by Deutsche Forschungsgemeinschaft (DFG 1321).

Competing interests

Alexander Meining is a consultant for OVESCO (Tübingen, Germany), the company that manufactures and distributes the device.

\section{References}

[1] Inoue $\mathrm{H}$, Endo $\mathrm{M}$, Takeshita $\mathrm{K}$ et al. A new simplified technique of endoscopic esophageal mucosal resection using a cap-fitted panendoscope (EMRC). Surg Endosc 1992; 6: 264-265

[2] Ono H, Kondo H, Gotoda T et al. Endoscopic mucosal resection for treatment of early gastric cancer. Gut 2001; 48: 225-229

[3] Oyama T, Yahagi N, Ponchon T et al. How to establish endoscopic submucosal dissection in Western countries. World J Gastroenterol 2015; 21: $11209-11220$

[4] Kwon C-I. Endoscopic submucosal dissection (ESD) training and performing esd with accurate and safe techniques. Clin Endosc 2012; 45: $347-349$

[5] de Melo SW, Cleveland P, Raimondo M et al. Endoscopic mucosal resection with the grasp-and-snare technique through a double-channel endoscope in humans. Gastrointest Endosc 2011; 73: 349-352

[6] Shimizu Y, Takahashi M, Yoshida T et al. Endoscopic resection (endoscopic mucosal resection/endoscopic submucosal dissection) for superficial esophageal squamous cell carcinoma: Current status of various techniques. Dig Endosc 2013; 25: 13-19

[7] Pimentel-Nunes P, Dinis-Ribeiro M, Ponchon T et al. Endoscopic submucosal dissection: European Society of Gastrointestinal Endoscopy (ESGE) Guideline. Endoscopy 2015; 47: 829-854

[8] Zizer E, Roppenecker D, Helmes F et al. A new 3D-printed overtube system for endoscopic submucosal dissection: first results of a randomized study in a porcine model. Endoscopy 2016; 48: 762 - 765

[9] Kantsevoy SV, Bitner M, Piskun G. New endoscopic platform for endoluminal en bloc tissue resection in the gastrointestinal tract (with videos). Surg Endosc 2016; 30: 3145 - 3151

[10] Schölvinck DW, Goto O, Bergman JJGHM et al. The efficacy of an endoscopic grasp-and-traction device for gastric endoscopic submucosal dissection: an ex vivo comparative study (with video). Clin Endosc 2015; 48: $221-227$ 